\title{
Simultaneous Intravitreal Ranibizumab and Dexamethasone Implant Administration at the Same Setting in Eyes with Severe Diabetic Macular Edema
}

\author{
Ali Osman Saatci*, Ziya Ayhan, Ceren Durmaz Engin \\ Department of Ophthalmology, Dokuz Eylul University, Izmir, Turkey \\ Email: "osman.saatci@yahoo.com
}

Received 14 April 2016; accepted 27 May 2016; published 31 May 2016

Copyright (C) 2016 by authors and Scientific Research Publishing Inc. This work is licensed under the Creative Commons Attribution International License (CC BY). http://creativecommons.org/licenses/by/4.0/

\begin{abstract}
Aim: To share our experience in eyes with severe DME (exhibiting serous retinal detachment or large cysts) treated with simultaneous intravitreal ranibizumab and dexamethasone implant administration at the same setting as the first treatment step. Subjects and Results: Five eyes of three patients with DME who were either treatment naive or relatively undertreated were presented in this report. As optical coherence tomography exhibited serous retinal detachment or severe cystoid edema with large cysts, intravitreal ranibizumab and dexamethasone implant were simultaneously employed at the same setting as the first treatment step in those eyes. Panretinal photocoagulation was also commenced bilaterally a week after the start of injections when at least one eye had retinal neovascularization. Subsequent treatments of intravitreal ranibizumab and/or dexamethasone implant were administered. Patients were followed up for seven, eight and 13 months respectively. All five eyes achieved a relative anatomic stability and experienced visual improvement at the end of follow-up. Conclusion: In some cases with severe DME with or without proliferative diabetic retinopathy, simultaneous intravitreal ranibizumab and dexamethasone implant administration at the same setting may be a better option to initiate the treatment over mono ranibizumab treatment. A randomized study comparing the mono anti-VEGF therapy and mono dexamethasone implant administration with simultaneous treatment may outline the place of this type of therapy in the treatment armamentarium of severe DME.
\end{abstract}

\section{Keywords}

Dexamethasone Implant, Diabetic Macular Edema, Optical Coherence Tomography, Ozurdex, Ranibizumab

\footnotetext{
${ }^{*}$ Corresponding author.
}

How to cite this paper: Saatci, A.O., Ayhan, Z. and Engin, C.D. (2016) Simultaneous Intravitreal Ranibizumab and Dexamethasone Implant Administration at the Same Setting in Eyes with Severe Diabetic Macular Edema. Open Journal of Ophthalmology, 6, 112-118. http://dx.doi.org/10.4236/ojoph.2016.62016 


\section{Introduction}

Diabetic macular edema (DME) can be classified into three morphological types as diffuse retinal thickening, cystoid macular edema and serous retinal detachment (SRD) with the help of optical coherence tomography (OCT) [1]. Presence of SRD was noted in up to 36.5\% of eyes with DME [2] and the prevalence of SRD was directly proportional to the severity of the diabetic retinopathy [3].

Inflammatory factors besides the VEGF play an important role in the pathogenesis of diabetic retinopathy [4]. Study of 27 aqueous humor cytokines in patients with type 2 diabetes with or without retinopathy demonstrated that VEGF, interleukin-1 $\beta$ (IL-1 $\beta$ ), interleukin 6 (IL-6), interleukin 8 (IL-8), monocyte chemoattractant protein-1 (MCP-1) and interferon induced protein 10 (IP-10) levels in the aqueous humor were increased in accordance with the severity of diabetic retinopathy [5]. In another study, IL-6 was found to be the pivotal factor associated with the presence of SRD in DME [2]. It was also shown that intravitreal bevacizumab injection could only reduce the aqueous VEGF levels whereas intravitreal triamcinolone acetonide injection could reduce not only the VEGF but also the IL-6, MCP-1, IP-10 and platelet derived growth factor AA (PDGF-AA) levels [6].

Therefore, it may be reasonable to administer intravitreal ranibizumab and dexamethasone implant simultaneously in eyes with severe DME in order to suppress both the VEGF and inflammatory cytokines at least at the initiation of pharmacological therapy. We hereby report five eyes of three patients that were treated with simultaneous intravitreal ranibizumab and dexamethasone implant at the same setting as the initial therapeutic procedure.

\section{Report of the Cases}

\subsection{Case 1}

A 51-year-old man with type 2 diabetes of eight known years of disease duration was examined by us for bilateral marked visual deterioration. He had also hypertension and microalbuminuria. On our examination, bestcorrected visual acuity was 4/10 in OD and 3/10 in OS. Slit-lamp evaluation was unremarkable bilaterally. Fundus examination and fluorescein angiography revealed severe macular edema OU and the presence of NVE in OD (Figures 1(a)-(d)). OCT examination demonstrated the presence of serous retinal detachment in OS and large cysts with a cystic cavity in OS (Figure 1(e) and Figure 1(f)). The option of simultaneous intravitreal ranibizumab and dexamethasone implant administration was explained to the patient as we felt that the visual outcome might be better over monotherapy. Both eyes underwent uneventful simultaneous intravitreal ranibizumab and dexamethasone implant administration one week apart at the same setting. A week later, panretinal photocoagulation together with macular laser was commenced and completed in three separate sessions. Three more bilateral ranibizumab injections were given in a timespan of four months. While the right eye received the fifth ranibizumab injection alone, left eye received once again simultaneous ranibizumab and dexamethasone implant simultaneously at the same setting due to recurrence of macular edema again with very large cysts. Approximately eight months after the initial presentation best-corrected visual acuity was 6/10 in OD and 4/10 in OS and both posterior poles looked relatively stable (Figures 2(a)-(d)).

\subsection{Case 2}

A 62-year-old woman with type 2 diabetes of six known years' duration was referred to us as she decided to have a second opinion after having laser photocoagulation elsewhere. On our examination, best-corrected visual acuity was $1 / 10$ in OD and counting fingers at 3 meters in OS. Slit-lamp examination demonstrated +2 nuclear sclerosis and +1 posterior subcapsular opacity OU. Intraocular pressure was $14 \mathrm{~mm} \mathrm{Hg}$ bilaterally.

Fundus examination revealed severe diabetic macular edema, macular exudation and NVE OU (Figures 3(a)-(d)). OCT delineated the presence of SRD OU (Figure 3(d) and Figure 3(e)). The therapeutic options were discussed with the patient and family. Upon receiving the consent, simultaneous intravitreal ranibizumab and dexamethasone implant were administrated one week apart. A week after the injections panretinal photocoagulation was commenced and completed in three sessions. Modified grid laser was also performed in addition to pharmacotherapy OU. Three more intravitreal ranibizumab injections were subsequently given in a monthly fashion. Five months after the start of injections, second dexamethasone implant was again administrated bilaterally a week apart. Almost a year after the initiation of therapy both eyes underwent uneventful phacoemulsification, intraocular lens implantation and $4 \mathrm{mg}$ intravitreal triamcinolone acetonide injection one week apart. 


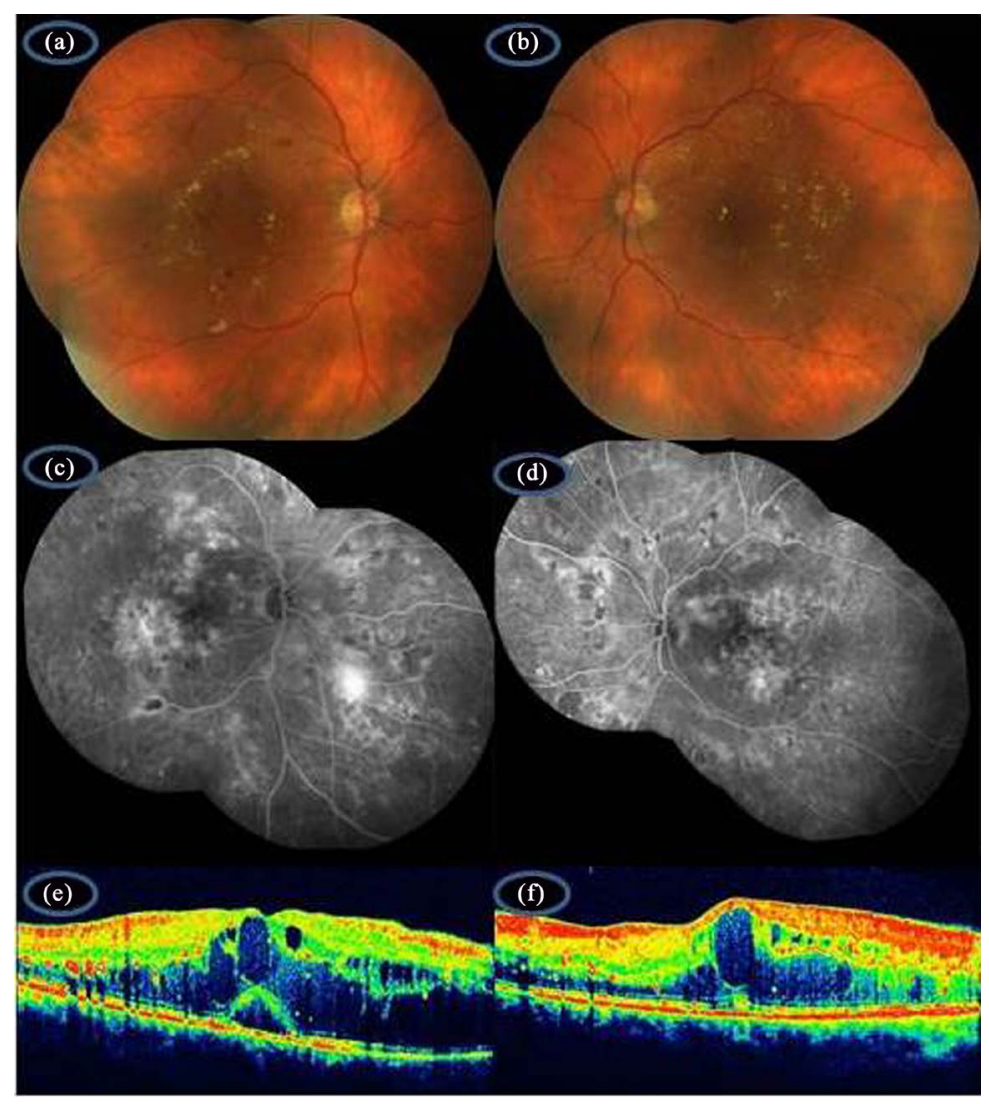

Figure 1. Composite color fundus pictures of the right (a) and the left eye (b) depicting the severe diabetic macular edema. Composite fluorescein angiographic picture of the right eye showing the diffuse macular leakage, leakage related to NVE nasal to the disc peripheric retinal capillary nonperfusion areas (c) and composite fluorescein angiographic picture of the left eye showing the diffuse macular leakage and retinal peripheric capillary nonperfusion areas (d) OCT exhibiting serous subretinal fluid with severe macular thickening in OD (e) and large retinal cysts with severe macular thickening in OS (f).

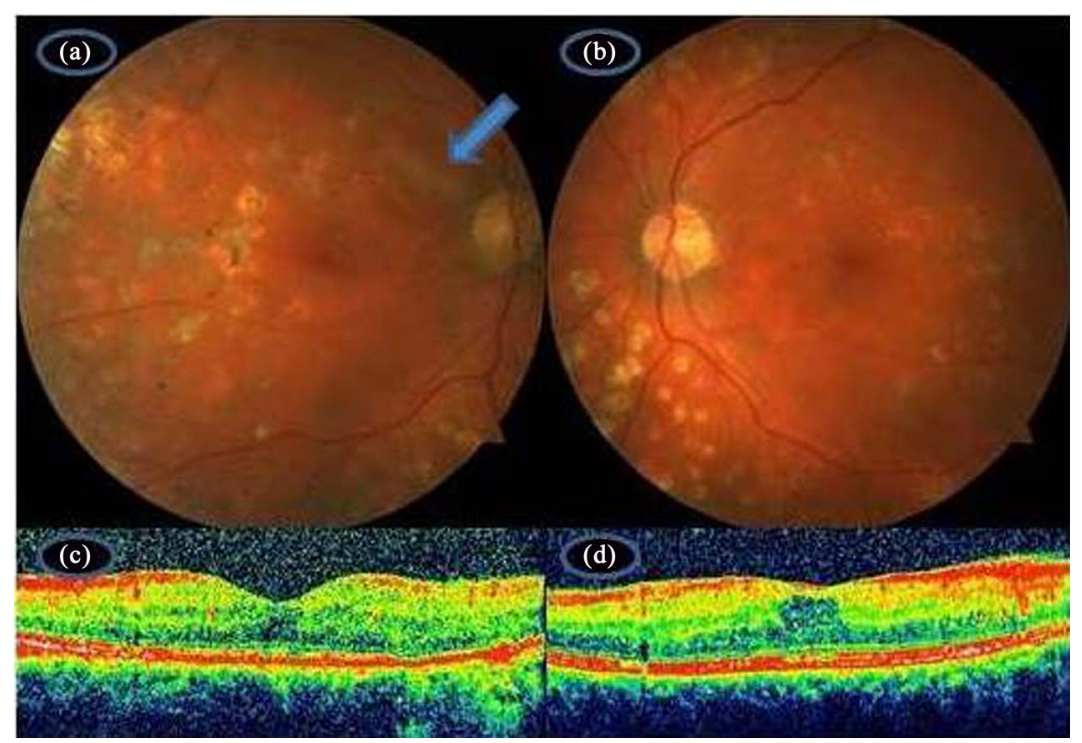

Figure 2. Color fundus picture of the right eye showing the stable looking posterior pole with a small residue of the dexamethasone implant (arrow) in OD (a) and color fundus picture of the left eye showing the relatively stable posterior pole with a few hard exudates (b). OCT scan of the right (c) and the left (d) eye demonstrating the relatively thinned macula with almost a normal foveal contour. 


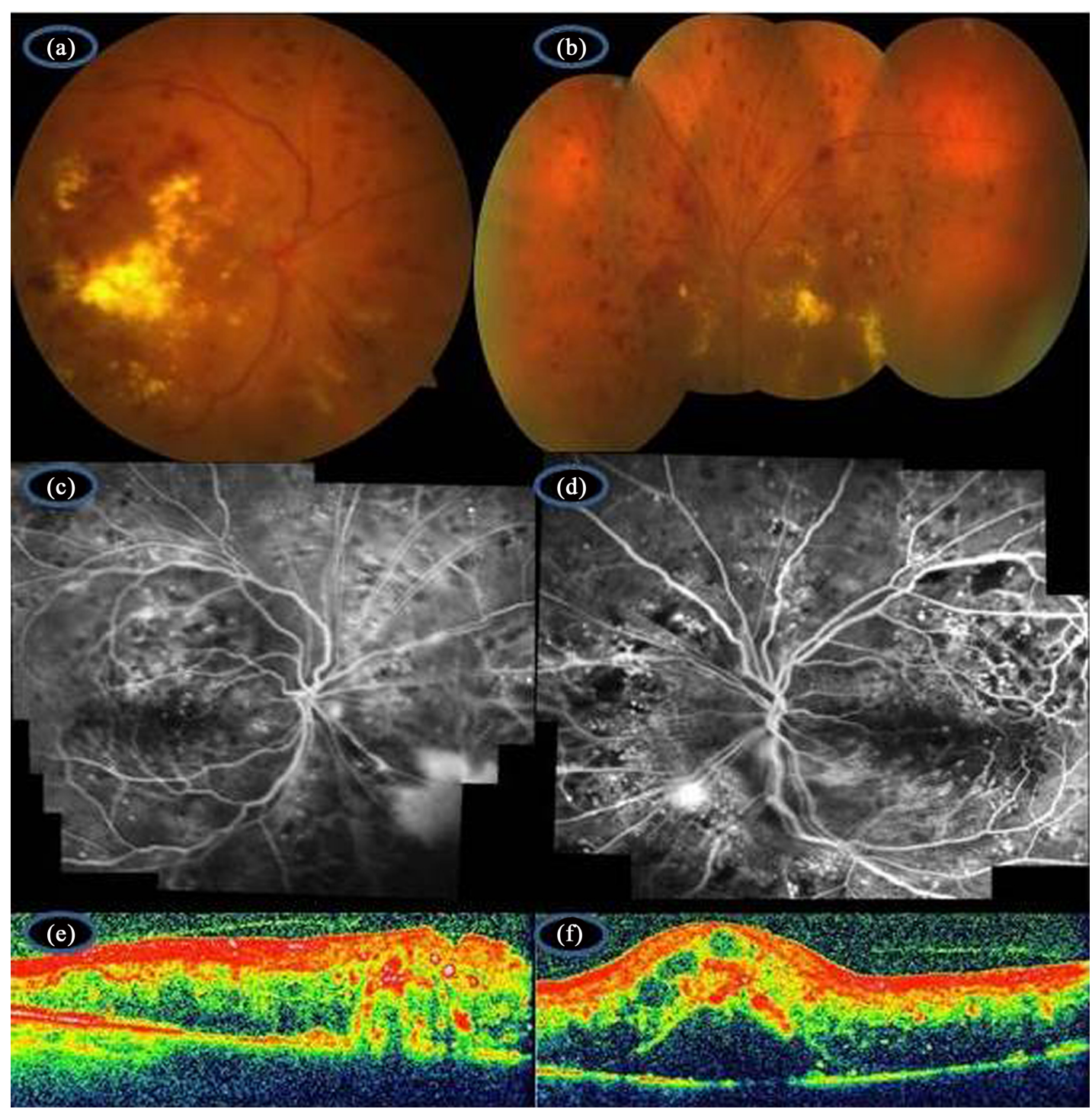

Figure 3. Composite color fundus pictures of the right (a) and the left eye (b) depicting the severe diabetic macular edema with extensive exudation. Composite fluorescein angiographic pictures of the right (c) and left (d) eye demonstrating the neovascularization and severe macular leakage. OCT scan delineating the severe foveal thickening in OD (e) and marked foveal thickening with serous subfoveal retinal fluid in OS (f).

Best-corrected visual acuity was 3/10 in OD and 2/10 in OS two weeks after the last cataract surgery and the retinopathy status looked much better (Figures 4(a)-(d)). Unfortunately the patient suffered a serious stroke and was unable to return for further treatment as she became hemiplegic.

\subsection{Case 3}

A 55-year-old woman with type 1 diabetes with a disease duration of 25 years was referred to us. Her past medical history revealed a heart bypass surgery and renal artery stenting. She had undergone bilateral laser photocoagulation elsewhere. On our examination, best-corrected visual acuity was 1/10 OU. Slit-lamp examination was unremarkable bilaterally. Intraocular pressure was $15 \mathrm{~mm} \mathrm{Hg}$ in both eyes. Fundus examination and fluorescein angiography demonstrated bilateral severe macular edema and NVE in OD (Figure 5(a) and Figure 5(b)). OCT demonstrated the serous retinal detachment in OD (Figure 5(c)) and retinal thickening in OS. Due to presence of serous retinal detachment, we elected to initiate the treatment with simultaneous intravitreal ranibizumab and dexamethasone implant administration in OD and mono ranibizumab therapy in OS. Following the 


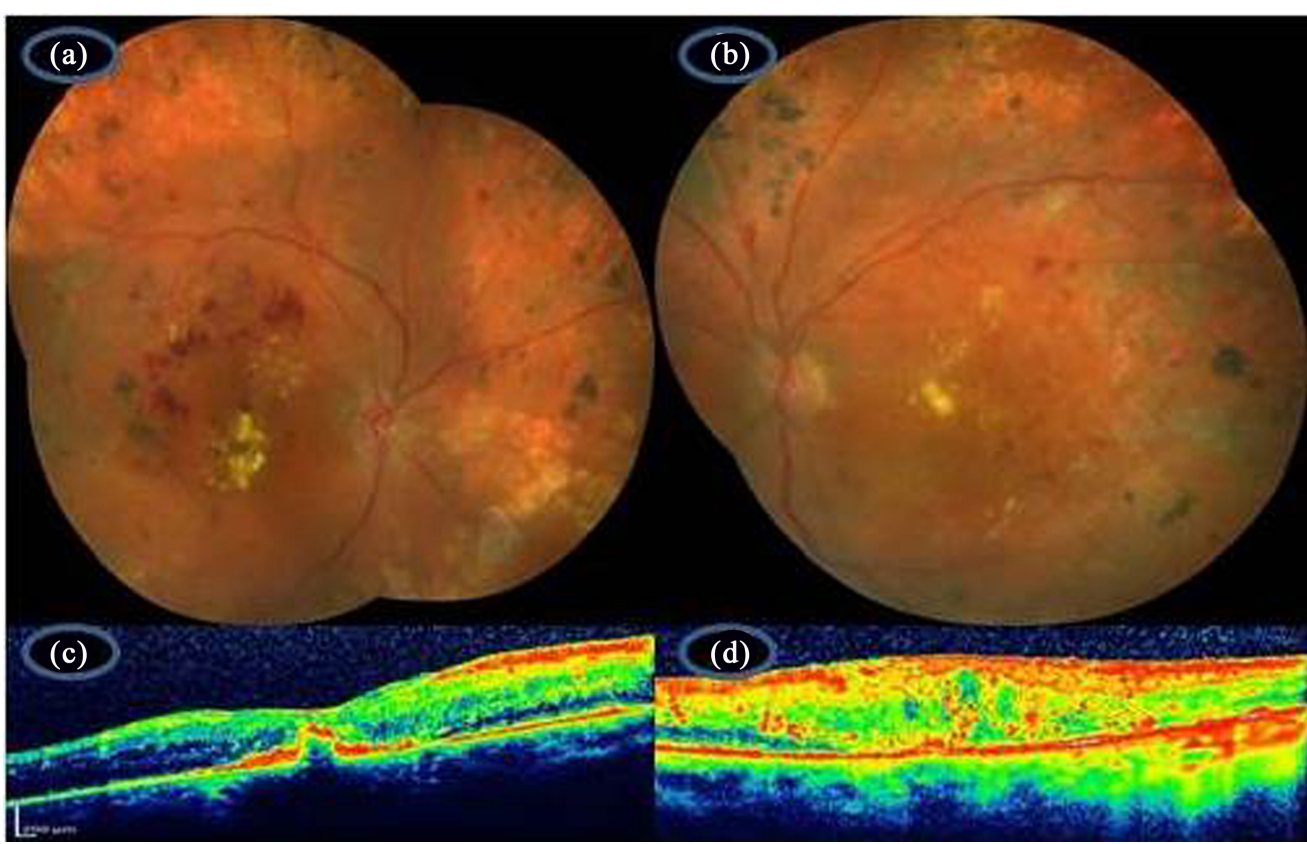

Figure 4. Composite color fundus pictures of the right (a) and left (b) eye showing the scattered laser scars 360 degree and markedly reduced macular hard exudates. OCT scan showing the relatively thinned macula with a small scar formation in OD (c) and relatively improved foveal contour in OS (d).

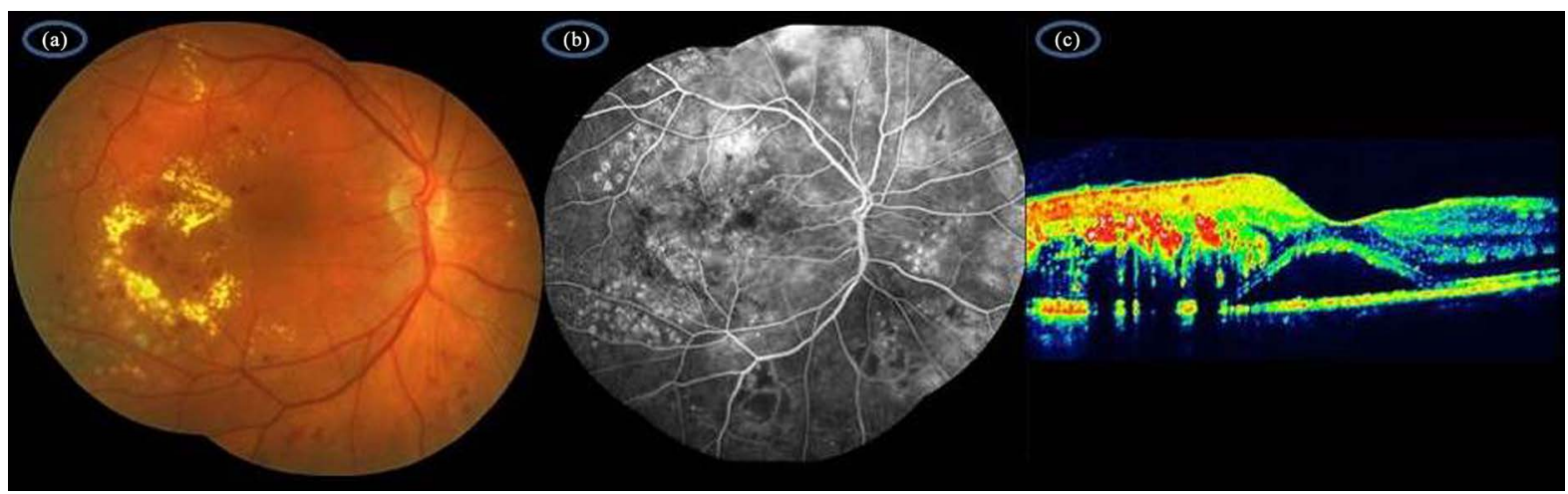

Figure 5. Right eye. Composite color fundus picture showing the severe circinate exudation temporal to the fovea (a), composite fluorescein angiographic frame demonstrating the severe macular leakage and leakage in association with NVE (b) and the OCT scan depicting the subfoveal serous fluid accumulation (c).

first injection panretinal photocoagulation was commenced and completed in three sessions. Four additional bilateral ranibizumab injection were given in a timespan of six months OU. Seven months after the start of therapy, best-corrected visual acuity was 3/10 in OD and 2/10 in OS with a significantly improved fundus appearance (Figure 6(a) and Figure 6(b)).

\section{Discussion}

Though anti-VEGF treatment is the gold standard for treating the center-involved DME, we believe that there is still room to make progress for obtaining better anatomic and visual results. In a very recently published posthoc analysis from Protocol I, 40\% of eyes were reported to have persistent macular thickening despite receiving of at least 4 intravitreal ranibizumab injections 24 weeks after the start of therapy [7]. Therefore, at least in some eyes with DME, simultaneous anti-VEGF and steroid treatment can be preferred over mono anti-VEGF therapy in order to suppress both the VEGF and inflammatory cytokines. A meta-analysis comparing the effect of intravitreal bevacizumab alone with combined bevacizumab and triamcinolone injection was recently published 


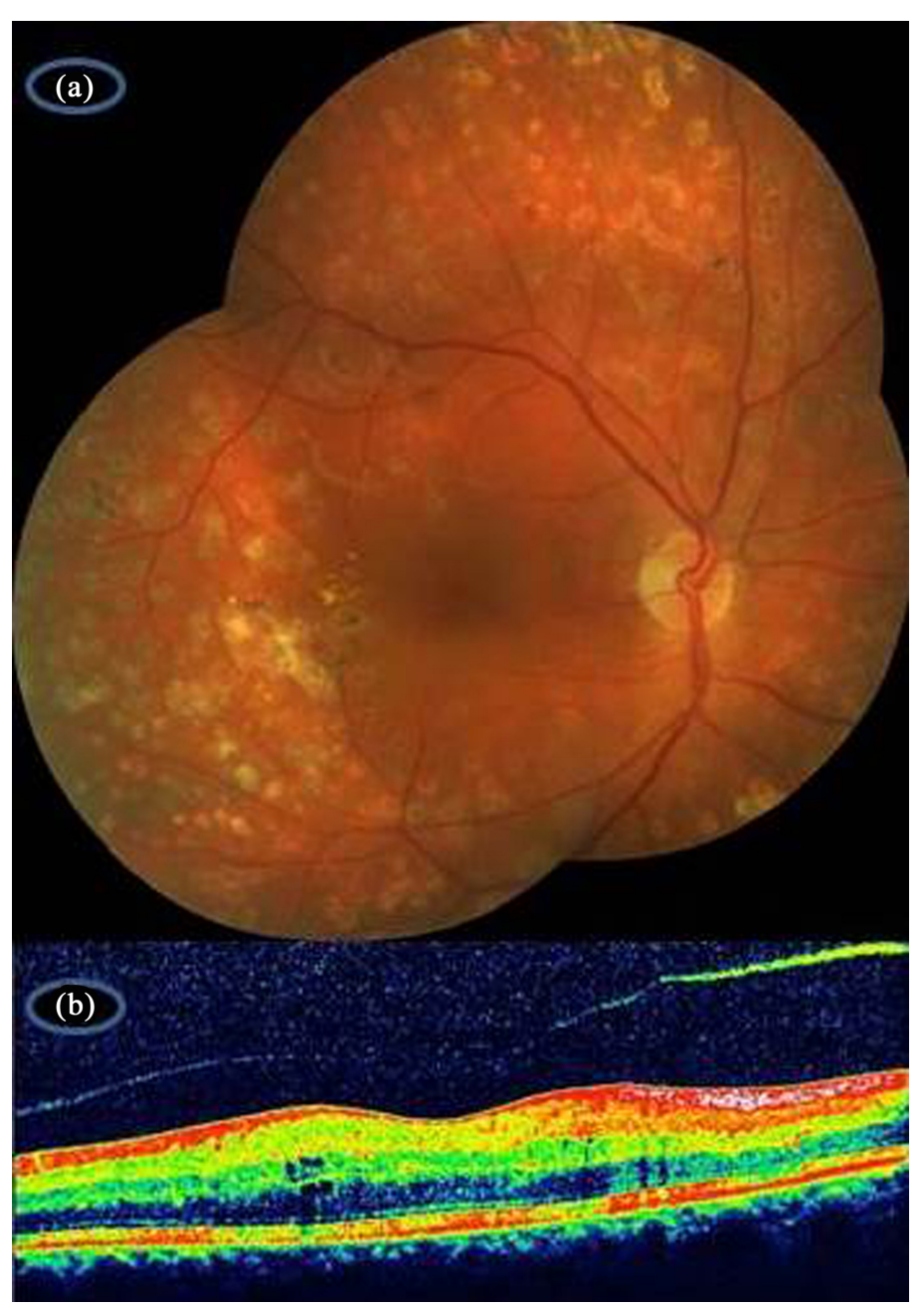

Figure 6. Right eye. Composite color fundus picture showing the almost dry macula with full panretinal photocoagulation (a); OCT scan showing the normal looking foveal contour (b).

and it was suggested that intravitreal treatment with bevacizumab alone and combined bevacizumab and triamcinolone acetonide administration resulted in similar visual improvement but to some degree the combined treatment protocol appeared to offer a marginal advantage over patients treated with only bevacizumab injection [8].

Clinicians still continue to elucidate which clinical clues present at the baseline examination are meaningful to predict the success of anti-VEGF monotherapy in eyes with DME. Some authors documented that morphological edema type might predict the therapeutic outcome and help the clinicians to choose the best treatment alternative [9]-[12]. In a group of 65 eyes of 48 patients treated with three monthly $1.25 \mathrm{mg}$ bevacizumab injections, the treatment effect was assessed according to baseline OCT pattern of the edema [11]. Eyes with SRD and cystoid macular edema responded less when compared to eyes with diffuse macular thickening in regard to central foveal thickening and visual outcome at the sixth month of treatment. Seo et al. [12] prospectively evaluated the outcome of intravitreal ranibizumab treatment in 55 eyes with DME based on the morphologic pattern of edema. Best-corrected visual acuity was significantly worse in eyes with SRD after 12 months than that of other types of macular edema. Moreover, disruption of the photoreceptor integrity at the baseline was more frequently observed in eyes with SRD. Thereby visual outcome was much poorer.

Nowadays, we intend to inject intravitreal ranibizumab and dexamethasone implant simultaneously at the same setting for the treatment of treatment-naïve or previously undertreated eyes with severe DME with or without proliferative diabetic retinopathy as the initial therapeutic approach. For the time being only ranibizumab is reimbursed for the DME treatment in Turkey. Aflibercept can also be combined with dexamethasone im- 
plant for the same purpose. However, only a prospective study will ascertain the place of simultaneous treatment with anti-VEGF agents and dexamethasone implant over mono anti-VEGF agents in eyes with severe DME at least for the kickoff of therapy.

\section{References}

[1] Otani, T., Kishi, S. and Maruyama, Y. (1999) Patterns of Diabetic Macular Edema with Optical Coherence Tomography. American Journal of Ophthalmology, 127, 688-693. http://dx.doi.org/10.1016/S0002-9394(99)00033-1

[2] Sonoda, S., Sakamoto, T., Yamashita, T., et al. (2014) Retinal Morphologic Changes and Concentrations of Cytokines in Eyes with Diabetic Macular Edema. Retina, 34, 741-748. http://dx.doi.org/10.1097/IAE.0b013e3182a48917

[3] Alkuraya, H., Kangave, D. and Abu El-Asrar, A.M. (2005) The Correlation between Optical Coherence Tomographic Features and Severity of Retinopathy, Macular Thickness, and Visual Acuity in Diabetic Macular Edema. International Ophthalmology, 26, 93-99. http://dx.doi.org/10.1007/s10792-006-9007-8

[4] Noma, H., Mimura, T., Yasuda, K. and Shimura, M. (2014) Role of Inflammation in Diabetic Macular Edema. Ophthalmologica, 232, 127-135. http://dx.doi.org/10.1159/000364955

[5] Dong, N., Xu, B., Chu, L. and Tang, X. (2015) Study of 27 Aqueous Humor Cytokines in Type 2 Diabetic Patients with or without Macular Edema. PloS One, 10, e0125329. http://dx.doi.org/10.1371/journal.pone.0125329

[6] Sohn, H.J., Han, D.H., Kim, I.T., et al. (2011) Changes in Aqueous Concentrations of Various Cytokines after İntravitreal Triamcinolone versus Bevacizumab for Diabetic Macular Edema. American Journal of Ophthalmology, 152, 686-694. http://dx.doi.org/10.1016/j.ajo.2011.03.033

[7] Bressler, S.B., Ayala, A.R., Bressler, N.M., et al. (2016) Persistent Macular Thickening after Ranibizumab Treatment for Diabetic Macular Edema with Vision İmpairment. JAMA Ophthalmology, 134, 278-285.

http://dx.doi.org/10.1001/jamaophthalmol.2015.5346

[8] Liu, X., Zhou, X., Wang, Z., et al. (2014) Intravitreal Bevacizumab with or without Triamcinolone Acetonide for Diabetic Macular Edema: A Meta-Analysis of Randomized Controlled Trials. Chinese Medical Journal, 127, 3471- 3476.

[9] Wu, P.C., Lai, C.H., Chen, C.L. and Kuo, C.N. (2012) Optical Coherence Tomographic Patterns in Diabetic Macula Edema Can Predict the Effects of İntravitreal Bevacizumab İnjection as Primary Treatment. Journal of Ocular Pharmacology and Therapeutics, 28, 59-64. http://dx.doi.org/10.1089/jop.2011.0070

[10] Kim, M., Kim, Y. and Lee, S.J. (2015) Comparison of Aqueous Concentrations of Angiogenic and Inflammatory Cytokines Based on Optical Coherence Tomography Patterns of Diabetic Macular Edema. Indian Journal of Ophthalmology, 63, 312-317. http://dx.doi.org/10.4103/0301-4738.158069

[11] Kim, M., Lee, P., Kim, Y., et al. (2011) Effect of İntravitreal Bevacizumab Based on Optical Coherence Tomography Patterns of Diabetic Macular Edema. Ophthalmologica, 226, 138-144. http://dx.doi.org/10.1159/000330045

[12] Seo, K.H., Yu, S.Y., Kim, M. and Kwak, H.W. (2016) Visual and Morphologic Outcomes of Intravitreal Ranibizumab for Diabetic Macular Edema Based on Optical Coherence Tomography Patterns. Retina, 36, 588-595. http://dx.doi.org/10.1097/IAE.0000000000000770 